\title{
Why comprehensive adoption of robotic assisted thoracic surgery is ideal for both simple and complex lung resections
}

\author{
Michael Mazzei, Abbas E. Abbas \\ Division of Thoracic Surgery, Department of Thoracic Medicine and Surgery, Lewis Katz School of Medicine at Temple University, Philadelphia, \\ PA, USA \\ Contributions: (I) Conception and design: AE Abbas; (II) Administrative support: AE Abbas; (III) Provision of study materials or patients: All authors; \\ (IV) Collection and assembly of data: All authors; (V) Data analysis and interpretation: All authors; (VI) Manuscript writing: All authors; (VII) Final \\ approval of manuscript: All authors. \\ Correspondence to: Abbas E. Abbas, MD, MS, FACS. Professor and Thoracic Surgeon in Chief, Department of Thoracic Medicine and Surgery, \\ Temple University Health System, Lewis Katz School of Medicine, 3401 N. Broad St., Suite C-100, Philadelphia, PA 19035, USA. \\ Email: abbas.abbas@temple.edu.
}

\begin{abstract}
Minimally invasive thoracoscopic surgical techniques have grown increasingly popular due to improved outcome measures compared to conventional rib-spreading thoracotomy. However, video-assisted thoracoscopic surgery (VATS) presents with unique technical challenges that have limited its role in certain cases. Here, we discuss our perspectives on the implementation of a successful robotic thoracic program. We will then present the case for how the adoption of robotic assisted thoracic surgery (RATS) provides the benefits of minimally invasive VATS while still retaining the technical finesse of bimanual articulating instruments and 3-dimensional imaging that is a universal component of any open surgery. We will also discuss how to overcome some of the perceived disadvantages to RATS in regard to the higher cost, lack of tactile feedback and potential safety concerns.
\end{abstract}

Keywords: Robotic; portal; lobectomy; emergent conversion

Submitted Jul 17, 2019. Accepted for publication Dec 30, 2019.

doi: $10.21037 /$ jtd.2020.01.22

View this article at: http://dx.doi.org/10.21037/jtd.2020.01.22

\section{Introduction}

For early-stage lung cancer, the gold standard of treatment remains surgical resection with removal of the draining lymphatic basin. While this has traditionally been performed through an open rib-spreading thoracotomy, minimally invasive techniques such as video-assisted thoracoscopic surgery (VATS) have gained increasing traction due to outcome advantages such as fewer overall complications $(1,2)$, less pain (3) and decreased length of stay $(4,5)$. Identification of these benefits has resulted in a phase shift in care such that the American College of Chest Physicians guidelines now describe VATS as the preferred means of intervention upon early-stage lung cancer (6) and the National Comprehensive Cancer Network non-small cell lung cancer guidelines suggest strong consideration of
VATS resection (7).

However, despite the potential clinical advantages of VATS resection over traditional rib-spreading thoracotomy for the management of early-stage lung cancer, it currently accounts for less than half of the lobectomies being performed (8). There are unique technical challenges associated with VATS thoracotomy that preclude its adoption in all situations. For one, VATS approaches necessitate a markedly different viewing angle compared to that of open thoracotomy-usually anterior to the hilum, requiring an anterior-to-posterior dissection with division of the fissure last. Additionally, VATS can be prohibitively challenging in the setting of large central tumors or tumors with significant hilar adenopathy (9). This is compounded by the suboptimal view through the thoracoscope, which as a two-dimensional image can 
limit depth perception and place further demands on the surgeon during difficult dissections. Furthermore, the need for rigid, straight instruments does not necessarily allow for easy or safe manipulation of the lung in the curved thoracic space.

Since the early 2000s, Robotic-assisted thoracic surgery (RATS) has represented a third option for major pulmonary resections (10). Since that time, the use of robotic approaches for thoracic surgery has grown exponentially in both the percentage of cases performed (estimated to be up to $20 \%$ of lobectomies in the US per year (11), and in the complexity of cases performed (now encompassing such varied procedures as lobectomy with bronchoplasty (12), chest wall resections, and very large tumors with hilar adenopathy (13). We feel that the robotic approach, as a natural extension of the minimally invasive thoracoscopic spectrum, contains all the advantages of VATS interventions when compared with the conventional open technique, while approximating to a large extent the technical finesse of an open operation. Here, we discuss our rationale for the adoption of the robotic approach as an increasingly desirable evolution in thoracic surgical technique.

\section{Robotic thoracic surgical outcomes}

In 2002, the first case series of pulmonary resections via a RATS approach were reported Melfi et al. This included five lobectomies, completed with a conversion rate of forty percent (10). This proof of concept was followed by a number of early adopters who pioneered the use of the robot in lobectomy as well as other thoracic procedures such as thymectomy, fundoplication, and esophageal dissection (14-16). Since that time, a number of cohort studies and case series have continued to demonstrate favorable clinical outcomes after robotic surgery.

In particular, modern case series and case control studies demonstrate excellent outcomes for roboticassisted pulmonary surgery in comparison to open thoracotomy. For example, in 2011 Cerfolio et al. examined a 168-person cohort and found decreased perioperative morbidity ( $27 \%$ vs. $38 \%$ ), and hospital length of stay compared to those undergoing conventional rib-spreading thoracotomy (13). This was confirmed in a 2014 case series of 394 RATS anatomic pulmonary resections by Nasir et al., in which thirty-day operative mortality was $0.25 \%$, and 90 -day mortality was $0.5 \%$ (which mirrors that of conventional rib-spreading thoracotomy for pulmonary resections). This demonstrated a conversion rate of $10.4 \%$, and a $9.6 \%$ rate of major morbidity; it was associated with excellent postoperative analgesia and sustainable financial metrics (17). More recently, in a 2017 matched analysis of 76 total patients, Darr et al. confirmed that patients undergoing robotic lobectomy have increased analgesia and a shorter length-of-stay compared to conventional thoracotomy (18).

While there are no prospective, randomized multicenter studies addressing the benefits of RATS versus VATS for pulmonary resections, there is a growing body of evidence to suggest that RATS may have improved outcomes by several metrics. In 2012, a case-control study by Louie et al. compared RATS versus VATS lobectomy operations matched by patient age, comorbidities, pulmonary function, and resection distribution. In this comparison, RATS outcomes were found to similar to VATS in terms of hospital length of stay, major morbidity, and conversion rates (19). A 2011 comparison of robot-assisted lobectomy versus VATS for lung cancer by Jang et al. highlighted a postoperative length of hospital stay reduction of over 3 days among RATS patients, with significantly favorable outcomes after RATS compared to VATS (20). A 2014 comparison of RATS with both VATS and thoracotomy using the STS database found a number of significant benefits to RATS relative to either conventional open or VATS surgery. This included a significant decrease in 30-day mortality, a hospital length-of-stay 2 days less than VATS and four days less than after open surgery, and generally favorable complications rates (21).

Primarily, the detractors of RATS thoracotomy highlight its increased cost compared to VATS that is not necessarily associated with improved outcomes. In a single-institution study comparing these two surgical modalities, Deen et al. identified a cost differential of $\$ 3,182$ more in RATS than VATS owing largely to the cost of robotic-specific supplies (22); at that same time a similar study by Paul et al. identified a cost differential of $\$ 4,708$. Swanson et al. compared hospital costs and clinical outcomes for VATS versus RATS, and identified a cost differential of between $\$ 2,992$ and $\$ 4,564$, without any differences in adverse events (23). A 2016 systematic review and meta-analysis of this issue concluded that RATS provides advantages over thoracotomy but appears to be no different than VATS, with an associated increased cost (24). As RATS technology is still in a phase of early adoption, it will be interesting to see if the differences in outcomes between VATS and RATS are more consistently seen as experience with RATS grows. 


\section{Learning curve of RATS pulmonary resection}

There is significant evidence to suggest that the primary reason for failure of universal VATS adoption is due to technical aspects of the operation compared to open thoracotomy. In a survey through the European Society of Thoracic Surgeons the single most common reason cited for avoiding VATS surgery in favor of an open approach was the perceived technical challenge of the procedure (25). The steep learning curve is one noted reason for this; for example, initial studies of the VATS lobectomy procedure identified a case requirement of at least fifty cases in the hands of experienced open surgeons (26). More recent reports continue to suggest comparatively poor outcomes in early cases with a requirement of nearly thirty cases prior to outcome equivalence, and an early caseload requirement of nearly forty VATS cases before a benefit in terms of hospital stay can be identified (27).

In comparison, robotic lobectomy appears to have a learning curve that is established after less than twenty cases. For example, Meyer et al. propose a learning curve time of $18 \pm 3$ cases based on operative times (28); Lee et al. suggest a range of $16 \pm 1$ (29). In some reviews, this learning curve has been truncated to as low as fourteen cases (30). This speed of skill acquisition is consistently highlighted to be due to operative factors which allow the robot to more closely approximate the comfort of open surgery (31).

\section{Implementing a successful RATS program}

While robotic thoracic surgery has the capacity to offer significant technical, ergonomic, and potentially outcomes benefits, it is important to note that with the implementation of robotic surgery comes several caveats. Robotic surgery, much like any surgical platform, is dependent significantly on fastidious team-wide understanding of the system and a careful plan for implementation. We have previously described our surgical technique for robotic lung resection $(32,33)$. We will highlight some "take-home" points that can facilitate the implementation of a successful robotic thoracic surgical program to ensure that the benefits of the platform are optimized, rather than stymied by suboptimal pre-, intra-, and postoperative decision making.

\section{Operating room efficiency}

As the robotic system's physical footprint is somewhat larger than that of traditional thoracoscopic platforms, a streamlined process is imperative in order to work smoothly within its physical confines. The complexity of the procedures demands significant training and ongoing maintenance in servicing of the entire surgical team including anesthesia. This may help to avoid inefficiencies that can make, operative and turnover time longer. In the operating room, a well-described and standardized protocol of bed and patient positioning, type of anesthesia care, monitoring, port placement, instrument choice, and team members streamlines the process.

\section{Device model}

It is important to understand the pros and cons of the robot model that is used for each procedure. The current DaVinci ${ }^{\odot}$ robotic system adds several advantageous features such as 360-degree rotation of the arms and higher camera definition. It allows for docking of the robot from the side of the patient instead of the head, freeing the patient's head for anesthesia to monitor and manipulate the endotracheal tube if necessary. It also provides for robotic vascular stapling which allows the main surgeon to retain control over the division of the hilar structures, as opposed to older models that required this most important function to be done by the bedside assistant.

\section{Three-vs. four-arm technique}

The decision regarding three or four robotic arms is a matter of surgeon's choice. The authors of this article espouse a four-arm technique ( 3 instrument ports plus camera which allows the console surgeon to both retract and perform bimanual dissection. This permits the bedside assistant to be more effective in keeping the field dry in addition to being able to remove specimens and introduce materials, e.g., sutures and gauze cigars without having to release the retraction of the lung to do so.

\section{Totally portal vs. portal plus utility incision}

This also is a matter of surgeon's preference. The recent AATS nomenclature statement differentiates the 2 approaches (robotic portal lobectomy (RPL) vs. robotic assisted lobectomy (RAL) when a utility incision is used (34). We prefer the totally portal approach although there is likely little if any difference in outcomes (35).

\section{Port placement (Figure 1)}

The decision regarding choice for port placement in 




Figure 1 Port placement. All 4 robotic ports in $8^{\text {th }}$ intercostal space. Assistant port in subcostal space, anterior to tip of the $11^{\text {th }}$ floating rib.

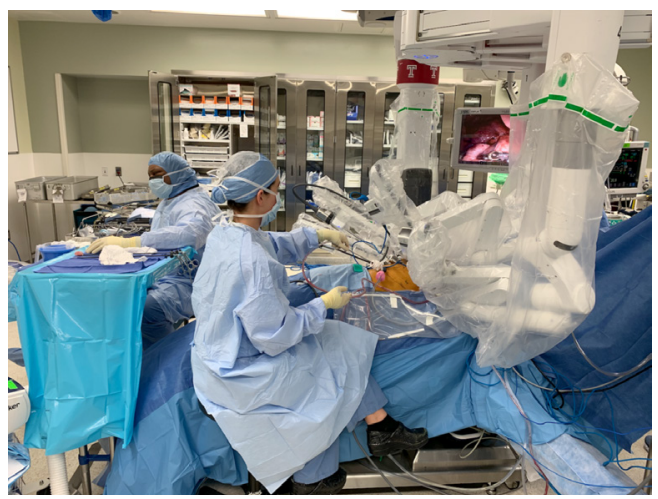

Figure 2 Side docking and bedside assistant.

multiple intercostal spaces versus one intercostal space is also a matter of team choice. The authors of this article prefer to place all of the robotic ports in the $8^{\text {th }}$ intercostal space when possible. We feel that this minimizes the risk of intercostal neuralgia for more than one rib space. We also prefer to place an assistant port in a subcostal fashion, through the insertion of the diaphragm onto the costal margin. Our rationale for this decision is to avoid specimen removal through an intercostal space, which also promotes intercostal nerve compression and neuralgia. While even very large specimens (e.g., pneumonectomies, large masses, chest wall resections) can be removed without the need for rib spreading in this manner. Indeed, it defeats the purpose to perform a minimally invasive portal lobectomy followed by a rib-spreading thoracotomy just to remove a large specimen. This technique, however, does require reinsertion of the diaphragm back onto the costal margin with permanent suture at the close of case.

\section{Body position and port sites}

The initial decision-making regarding patient positioning and port sites are critically important elements for this procedure. Once the robot is docked changes in patient positioning and port placement can be prohibitively difficult. A clear understanding of the angles required for free movement of the robotic arms, as well as careful attention to the clearance provided by the relationships of the ports to their target anatomy and to one another is imperative and requires experience. We prefer to place the patient in lateral decubitus position with maximal flexion of the table. We side-dock the patient cart leaving the head toward the anesthesia team (Figure 2).

\section{The surgical assistant}

Robotic thoracic surgery may be either facilitated or hampered by the degree of cooperation between the console surgeon and the bedside assistant. Because the primary surgeon is away from the patient's side, it is the bedside assistant's responsibility to lead the surgical team and alert the console surgeon to potential problems. The role of the assistant is especially critical during the occurrence of an emergent conversion, when the assistant must be able to handle the situation nearly independently while the console surgeon prepares to re-enter the surgical field (11) (Figure 2).

\section{Preoperative and postoperative patient care}

As any experienced surgeon knows, successful surgical outcomes are just as dependent on the preoperative preparation and postoperative management as they are on surgical technique. A well-run system must include provisions for management of the patient and surgical team at all phases of the operation. For example, in our institution as well as many other successful programs significant attention is paid to patient education prior to the procedure (36). Preoperative training on breathing, coughing and expectoration after surgery, and setting of surgical expectations can improve the patient experience as well as outcomes. Postoperatively, enhanced recovery pathways such as early ambulation, appropriate pain management and aggressive physical therapy protocols, as well early discharge when possible, all contribute to better outcomes.

\section{Benefits of RATS for anatomical lung resection}

Robotic surgery carries with it a number of technical 
benefits that can provide real advantages during challenging dissections when compared to traditional endoscopic surgery, while still preserving the decreased tissue trauma associated with that modality. The following are a number of reasons why we feel that RATS surgery provides a superior operative experience compared to traditional VATS minimally invasive approaches.

\section{Fully "wristed" instruments}

VATS instruments are typically long and rigid, allowing few options in terms of articulation. In comparison, the fully articulating, opposable robotic instruments have 7 degrees of freedom and thus can be maneuvered with essentially all the range of motion of the human wrist. This is particularly important when dissecting the pulmonary vessels or extirpating mediastinal lymph nodes, when careful bimanual articulating dissection based on sound surgical technique becomes of utmost importance.

\section{Decreased fulcrum pivoting}

The placement of VATS instrument or sometimes multiple instruments through a fixed trocar in the chest wall act as a fulcrum at the incision. In this situation, the surgeon's motor control system faces mechanical constraint at the incision point which poses challenges regarding the inversion and scaling of movements, as well as an altered sensation of haptic forces due to mechanical pressure and friction at the incision point (where the intercostal nerve resides). In comparison, RATS instruments have a pivot at the wrist of the instrument deep inside the surgical field. Although the fulcrum effect is not completely eliminated, the robotic port itself remains relatively fixed and is stationary for longer periods than in VATS. We and others hypothesized that the lack of torque and pressure shifts upon the thoracic trocar may cause less trauma to the associated intercostal nerves, with concomitant decreases in postoperative intercostal neuralgia $(37,38)$.

\section{A high-resolution magnified and binocular view}

The two-dimensional images of VATS can lead to challenges in depth perception, preventing the surgeon from instinctively judging the necessary maneuvers needed for difficult dissections. In contrast, RATS images are high-definition, and the binocular camera view provides a three-dimensional image with excellent perceived depth perception, comparable to naked eye vision. In addition, the image in RATS is also, magnifiable up to tenfold allowing clear visualization of small vessels and nerves that may otherwise not be noticed by other modalities. This is especially important in anatomical segmentectomy and subsegmentectomy (39).

\section{Mechanical sensitivity}

The sensitivity and scaling of the speed of the instruments is also modifiable and is coupled with further surgical aids such as tremor elimination features. The visual and mechanical precision provided by these features is especially suited to surgery in the pleural cavity, where fine dissection in a confined space is necessary.

\section{Physical strain reduction}

Traditional open surgery requires standing erect with the neck flexed, while often wearing heavy loupes. VATS requires standing and looking upwards at monitors. Performing long tedious operations, especially operating in the close multiple cases, can lead to ergonomically mediated chronic strain upon the operator. In contrast, a robotic procedure is performed in the seated position within an ergonomically optimized console potentially leading to a significant reduction of physical strain in the surgeon (40).

\section{Camera control}

In VATS, the operator must either relegate camera control to an assistant, must sacrifice a two-handed approach in order to manipulate the camera, or must work within a relatively static field using thoracoscopic camera holder devices. In contrast, the main surgeon retains control of the camera during robotic surgery and can easily constantly reposition it during the procedure for an optimal view. The surgical viewing angle is also top down, not from the side, much as we are used to in an open field. The surgeon has an instrument on each side of the camera which is a natural surrogate for the view in open surgery, with a hand on either side of the visual field. This completes the optical illusion of operating through a thoracotomy (31).

\section{Potential for teaching and training}

A double console allows two surgeons to operate 

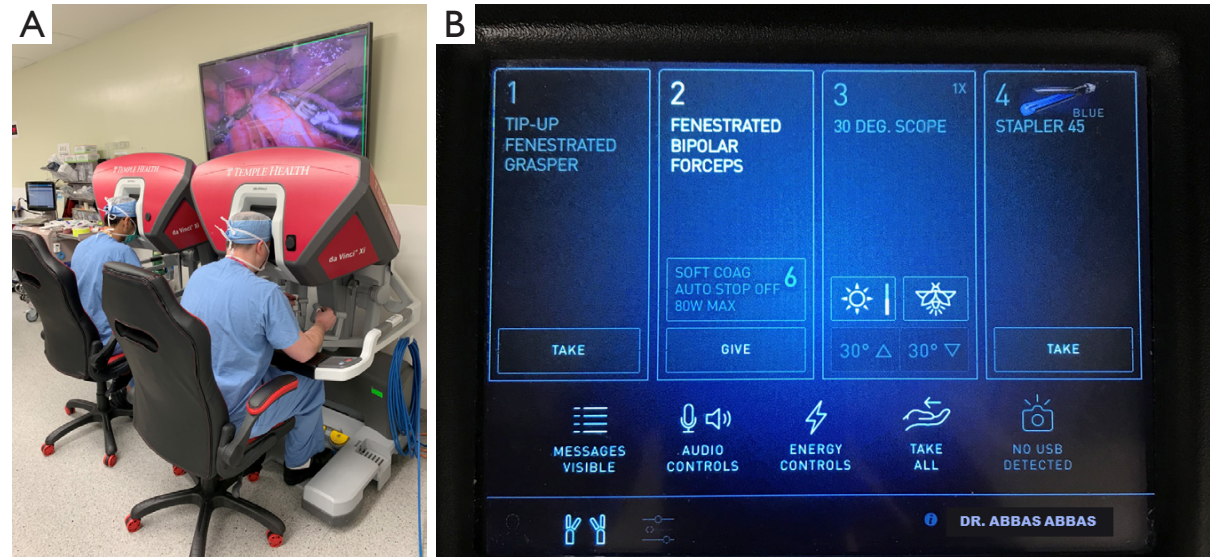

Figure 3 Using the double console. (A) The dual console allows trainees to have the same experience as the primary surgeon; (B) console control allows easy handoff of any or all of the instruments to either of the console surgeons.

simultaneously. This allows trainees to see the operative field exactly as the primary surgeon perceives it. In fact, this sharing of the exact same view is unique to robotics. In both open and VATS surgery, the surgeon is on the opposite side of at least one of the assistants thus creating a different perspective of the image for each of them. At the console, both surgeons see the same image from the same angle. A double console also provides the senior surgeon with the ability to point out anatomic features and guide the trainee within the operative field via console display markers, as well as to take over or switch control at any time. In fact, much like in open surgery, both surgeons can manipulate different instruments simultaneously. In addition, several simulator models have been developed that can enhance the ability of trainees to master this technology. These features allow for trainees to improve their abilities in an environment almost identical to actual live surgery thanks to the advances in digital simulation, where many of these models have become quite realistic. Again, this is another advantage unique to robotics which is inherently a digital platform (Figure 3).

\section{Ability to incorporate near infrared (NIR) autoflourescent imaging}

The current Da Vinci system incorporates a NIR emitter in the endoscopic camera. When indocyanin green (ICG), an injectable dye, is exposed to NIR light, it emits a fluorescent green image (Figure 4). Our group has previously published the first study on performing electromagnetic navigational bronchoscopic localization (ENBL) of indeterminate nodules followed by robotic wedge resection. Using this technique, Fifty-three of 54 indeterminate small, deep and subsolid nodules were successfully identified within the wedge resected specimen ( $98.1 \%$ accuracy). This obviated the frequent need in such cases for larger incisions or larger resections (41).

\section{Applicability in complex thoracic operations}

Traditionally VATS has been advocated mainly for early lung cancer. In fact, authors have advised against this technique for locally advanced tumors or when calcified nodes are identified (9). Although several studies have described VATS for major complex procedures, these are done mainly in a few highly specialized centers. On the other hand, it is the authors' opinion that the robotic approach carries the same indications as for open thoracotomy. Our group schedules all cases of lung resection for RATS, regardless of size of tumor, extent of resection, chest wall invasion or expected need for bronchoplastic or arterioplastic procedures. Our conversion rate has been below $2 \%$ and is mainly for severe adhesions. When indicated, we routinely perform RATS for complex lung resections including bronchial sleeve resections, arterial sleeve resections, pneumonectomy, possible mediastinal invasion, chest wall resections and even certain Pancoast tumors. In reviewing our outcomes, the number of complex procedures started and completed robotically was 106 of 1,408 robotic anatomical lung resections $(7.5 \%)$ The conversion rate for this group of complex resections was $4.7 \%$ (none for bleeding) while the $\mathrm{R} 0$ resection rate was $98.1 \%$ (Table 1). 

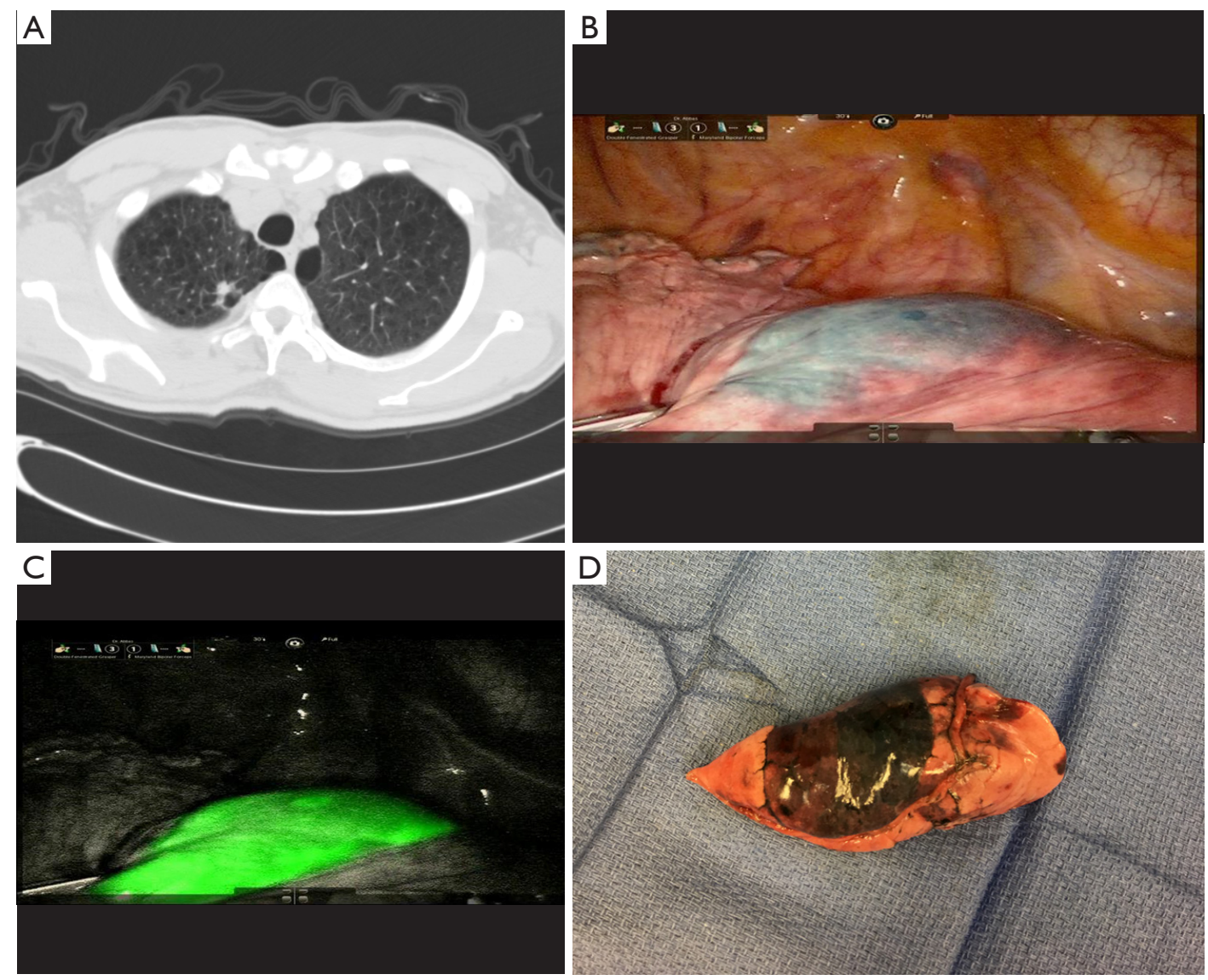

Figure 4 Intraoperative electromagnetic navigational bronchoscopic localization (ENBL) for indeterminate lung nodules. (A) Chest CT showing small indeterminate nodule; (B) right upper lobe nodule after ENBL with methylene blue and indocyanine green (ICG); (C) firefly mode clearly demarcates nodule; (D) small sized wedge resection along localization dye.

\section{Applicability for sublobar resections}

There is mounting evidence of the role of anatomical segmentectomy for early cancerous tumors of the lung including small or subsolid malignant nodules $(42,43)$. Thanks to magnification and motion scaling a surgeon is able to easily identify the tiny segmental and even subsegmental anatomy of the lung with ease and dexterity. In addition, we routinely employ NIR autoflourescence to identify the segmental margin for stapled division. ICG is given intravenously upon division of the segmental artery, vein and bronchus, and then, using NIR we are able to easily see the junction between the nonperfused target segment and perfused remaining lobe (Figure 5).

\section{Applicability in morbidly obese patients (Figure 6)}

In these patients, the robotic system provides unique advantages. Once the ports are placed, the console view does not differ at all as the robotic arms can easily adjust to the extra weight and resistance that may be met at the level of the entry point. Both VATS and thoracotomy on the other hand may be extremely difficult in these patients, requiring longer incisions and operating time.

\section{Continuing advances in robotic surgery}

The robotic surgical system has become a platform for ongoing technological development. Examples include a single port (SP) system (Intuitive Surgical, Inc., Sunnyvale, CA, USA), already introduced which includes 3 fully wristed instruments and an articulating camera, all through a $3 \mathrm{~cm}$ port. This has been FDA approved for urology and applications for other fields are in progress. In the near future, with the entrance of several new robotic surgery manufacturers onto the market, a number of technological 
Table 1 List of complex totally robotic lung resections

\begin{tabular}{|c|c|c|c|}
\hline Procedure & $\mathrm{N}$ & Notes & Complications \\
\hline Bronchial sleeve resection & 22 & RUL: 14, LUL 6, SS 2 & $\begin{array}{l}\text { Death: } 1 \text { (delayed BAF } \\
18 \text { months post-repair); } \\
\text { pneumonia: } 1 \text {; AMI: } 1 \text {; A.fib: } 6\end{array}$ \\
\hline Chest wall with anatomical lung resection & 10 & 2 with left pneumonectomy and 2 with Pancoast tumors & A.fib: 3 \\
\hline Pancoast tumor & 2 & $\begin{array}{l}\text { Robotic lobectomy and rib resection. Anterior } \\
\text { cervicomanubriotomy if vascular reconstruction is } \\
\text { necessary }\end{array}$ & \\
\hline
\end{tabular}

A.fib, atrial fibrillation; AMI, acute myocardial infarction; ARDS, adult respiratory distress syndrome; BAF, bronchoarterial fistula.
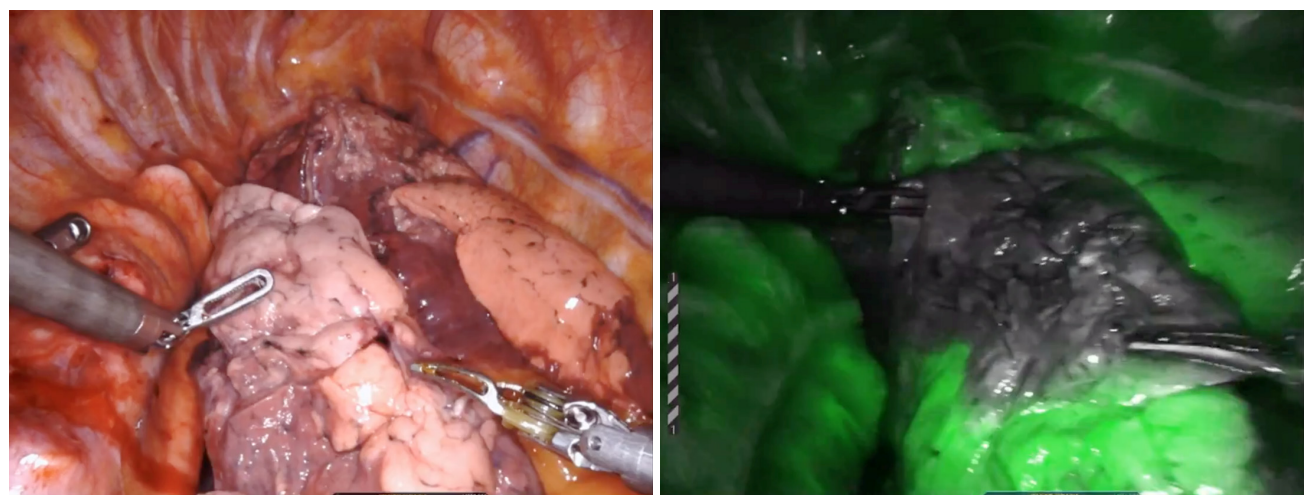

Figure 5 Using indocyanine green (ICG) pulmonary angiography during anatomical segmentectomy. (A) Normal view of right lower lobe after division of segmental pulmonary arterial branches to superior segment (S6); (B) view with Firefly mode after IV injection of 3 cc ICG clearly demarcates the margin of the non-perfused superior segment (S6).

innovations are likely to follow. Prototypes for haptic feedback will provides surgeons with tactile sensations similar to that experienced during open surgery. Systems for image overlaying, would allow the operating team to superimpose a reconstructed 3-dimensional study (such as a CT scan or MRI) over the real-time surgical image in order to be able to identify important anatomical structures and target pathology below the pleural surface (44).

\section{Disadvantages of robotic thoracic surgery}

Despite the above mentioned benefits, certain criticism may be directed to the robotic platform. These disadvantages include the following:

\section{Higher cost of robotic procedures}

Numerous publications comparing RATS to VATS have described the higher direct costs of lobectomy by RATS $(23,45)$. In an ongoing study at our institution on the impact of introducing RATS in an academic institution, high acuity services especially Thoracic Surgery was found to drive a higher contribution margin than other services. When other costs associated with hospital length of stay 

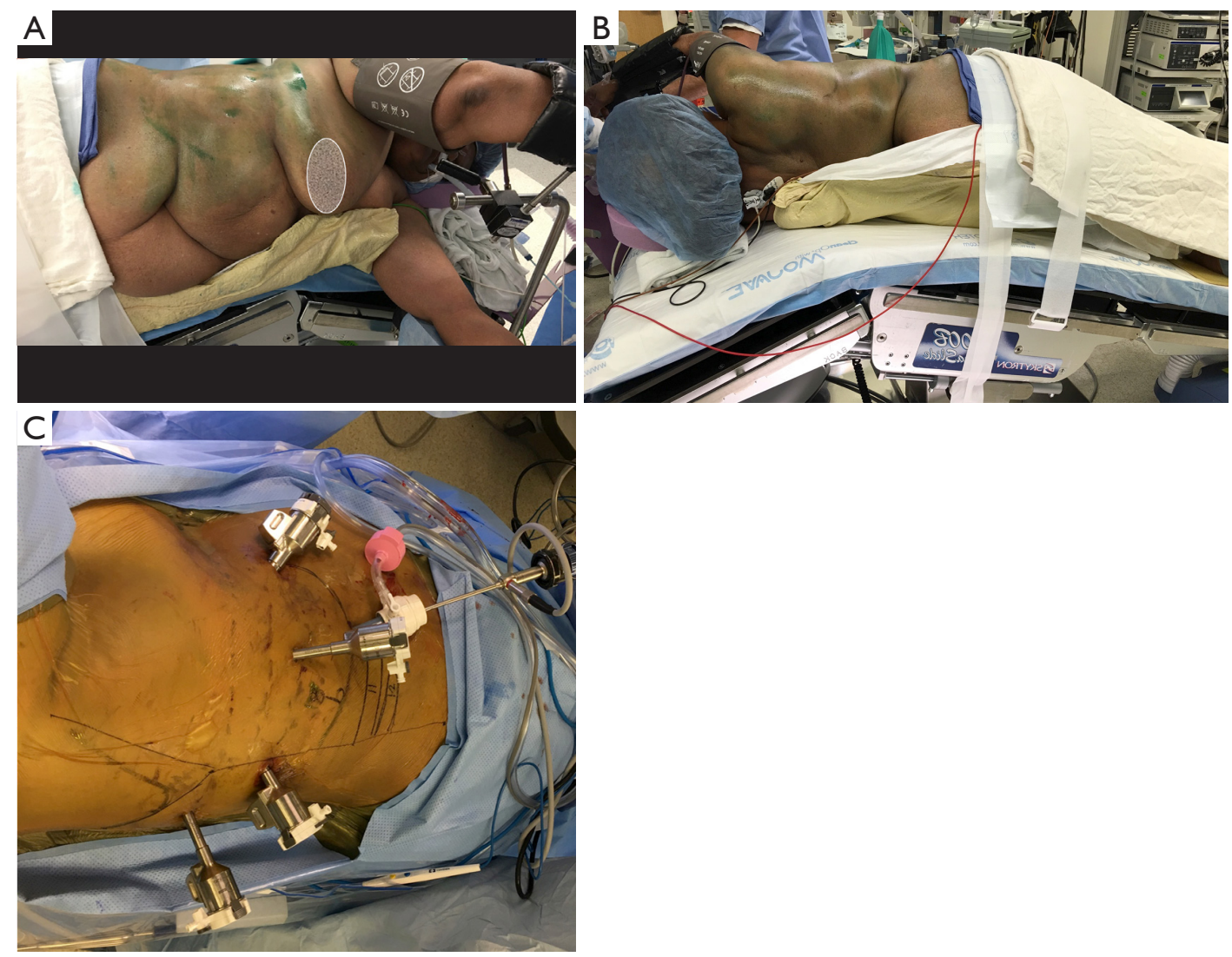

Figure 6 Benefit of RATS in obese patients. Same patient position and port placement regardless of BMI. (A) Front view; (B) back view; (C) port placement.

and complications are low, RATS can be associated with a significant cost saving to the institution.

\section{Safety concerns}

With RATS, the surgeon is not at the bedside and not even sterile. In addition, the surgeon has no "direct" contact with the organ he/she is operating upon. It is therefore important to regularly review emergency scenarios with the surgical team. Clear roles should be defined for each individual in the case, so that when it is necessary the team is prepared to work efficiently and in tandem. The authors review these roles before each case during a preprocedural "timeout," where the roles of calling for anesthesiologic assistance, obtaining blood, undocking the robot, and either holding tamponade or initiating a thoracotomy are assigned to the anesthesiologist, circulator, scrub nurse, and bedside assistant respectively (Table 2). While rare, conversion is best perceived as an expectation rather than a surprise for any busy thoracic program (31).

\section{Lack of tactile feedback}

Studies have shown that absence of haptic feedback can lead to both excessive and inadequate forces being applied to tissue during robotic surgery, which lead to increased tissue injury and inappropriate suture handling (46). However, many experienced robotic surgeons compensate for the lack of haptic feedback by becoming highly attuned to visual cues such as tissue deformation to act as surrogates for tension and force (47).

\section{Conclusions}

As a surgical platform, RATS combines the benefits of a minimally invasive technique with the technical and visual features that mimic open surgery. We have seen that with experience, RATS is an effective modality for even advanced 
Table 2 Preprocedural checklist timeout for emergency conversion

\begin{tabular}{ll}
\hline Job title & What to do \\
\hline Anesthesiologist & (I) Call for anesthesia support; (II) avoid reventilating the lung \\
Circulating nurse & $\begin{array}{l}\text { (I) If thoracotomy instruments not already open do that first; (II) obtain blood in the room; (III) notify blood bank } \\
\text { to stay } 4 \text { units ahead of whatever is released }\end{array}$ \\
Bedside assistant & $\begin{array}{l}\text { Depends on the situation: (I) if holding pressure on a bleeding vessel then continue to do that and do not } \\
\text { assist in anything else; (II) if control is lost then proceed with emergent thoracotomy (at } 5^{\text {th }} \text { intercostal space) }\end{array}$ \\
Scrub nurse or technician & $\begin{array}{l}\text { (I) Leave gown and glove on side of cart for surgeon to self-gown; (II) do not remove or switch off camera; (III) } \\
\text { undock only the arms that the console surgeon specifies; (IV) have thoracotomy instruments open; (V) have } \\
\text { bedside instrument-release wrench available }\end{array}$ \\
Console surgeon & (I) Gown and glove oneself; (II) call a colleague to assist; (III) keep calm and carry on
\end{tabular}

and complex cases such as sleeve resections, resection of large tumors or tumors with chest wall involvement, sizeable resections such as bilobectomy or pneumonectomy, and operating in a hostile surgical field where extensive adhesions or adenopathy would otherwise make for a treacherous dissection.

\section{Acknowledgments}

None.

\section{Footnote}

Conflicts of Interest: The authors have no conflicts of interest to declare.

Ethical Statement: The authors are accountable for all aspects of the work in ensuring that questions related to the accuracy or integrity of any part of the work are appropriately investigated and resolved.

\section{References}

1. Paul S, Altorki NK, Sheng S, et al. Thoracoscopic lobectomy is associated with lower morbidity than open lobectomy: a propensity-matched analysis from the STS database. J Thorac Cardiovasc Surg 2010;139:366-78.

2. Villamizar NR, Darrabie MD, Burfeind WR, et al. Thoracoscopic lobectomy is associated with lower morbidity compared with thoracotomy. J Thorac Cardiovasc Surg 2009;138:419-25.

3. Nagahiro I, Andou A, Aoe M, et al. Pulmonary function, postoperative pain, and serum cytokine level after lobectomy: a comparison of VATS and conventional procedure. Ann Thorac Surg 2001;72:362-5.

4. Atkins BZ, Harpole DH Jr, Mangum JH, et al. Pulmonary segmentectomy by thoracotomy or thoracoscopy: reduced hospital length of stay with a minimally-invasive approach. Ann Thorac Surg 2007;84:1107-12; discussion 1112-3.

5. Nomori H, Horio H, Naruke T, et al. What is the advantage of a thoracoscopic lobectomy over a limited thoracotomy procedure for lung cancer surgery? Ann Thorac Surg 2001;72:879-84.

6. Howington JA, Blum MG, Chang AC, et al. Treatment of stage I and II non-small cell lung cancer: Diagnosis and management of lung cancer, 3rd ed: American College of Chest Physicians evidence-based clinical practice guidelines. Chest 2013;143:e278S-e313S.

7. Ettinger DS, Wood DE, Aisner DL, et al. Non-Small Cell Lung Cancer, Version 5.2017, NCCN Clinical Practice Guidelines in Oncology. J Natl Compr Canc Netw 2017;15:504-35.

8. Blasberg JD, Seder CW, Leverson G, et al. Video-Assisted Thoracoscopic Lobectomy for Lung Cancer: Current Practice Patterns and Predictors of Adoption. Ann Thorac Surg 2016;102:1854-62.

9. McKenna RJ Jr, Houck W, Fuller CB. Video-assisted thoracic surgery lobectomy: experience with 1,100 cases. Ann Thorac Surg 2006;81:421-5; discussion 425-6.

10. Melfi FM, Menconi GF, Mariani AM, et al. Early experience with robotic technology for thoracoscopic surgery. Eur J Cardiothorac Surg 2002;21:864-8.

11. Abbas AE. Surgical Management of Lung Cancer: History, Evolution, and Modern Advances. Curr Oncol Rep 2018;20:98.

12. Cerfolio RJ. Robotic sleeve lobectomy: technical details and early results. J Thorac Dis 2016;8:S223-6.

13. Cerfolio RJ, Bryant AS, Skylizard L, et al. Initial 
consecutive experience of completely portal robotic pulmonary resection with 4 arms. J Thorac Cardiovasc Surg 2011;142:740-6.

14. Bodner J, Wykypiel H, Wetscher G, et al. First experiences with the da Vinci operating robot in thoracic surgery. Eur J Cardiothorac Surg 2004;25:844-51.

15. Park BJ, Flores RM, Rusch VW. Robotic assistance for video-assisted thoracic surgical lobectomy: technique and initial results. J Thorac Cardiovasc Surg 2006;131:54-9.

16. Rea F, Marulli G, Bortolotti L, Feltracco P, et al. Experience with the "da Vinci" robotic system for thymectomy in patients with myasthenia gravis: report of 33 cases. Ann Thorac Surg 2006;81:455-9.

17. Nasir BS, Bryant AS, Minnich DJ, et al. Performing robotic lobectomy and segmentectomy: cost, profitability, and outcomes. Ann Thorac Surg 2014;98:203-8; discussion 208-9.

18. Darr C, Cheufou D, Weinreich G, et al. Robotic thoracic surgery results in shorter hospital stay and lower postoperative pain compared to open thoracotomy: a matched pairs analysis. Surg Endosc 2017;31:4126-30.

19. Louie BE, Farivar AS, Aye RW, et al. Early experience with robotic lung resection results in similar operative outcomes and morbidity when compared with matched video-assisted thoracoscopic surgery cases. Ann Thorac Surg 2012;93:1598-604; discussion 1604-5.

20. Jang HJ, Lee HS, Park SY, et al. Comparison of the early robot-assisted lobectomy experience to video-assisted thoracic surgery lobectomy for lung cancer: a singleinstitution case series matching study. Innovations (Phila) 2011;6:305-10.

21. Farivar AS, Cerfolio RJ, Vallieres E, et al. Comparing robotic lung resection with thoracotomy and videoassisted thoracoscopic surgery cases entered into the Society of Thoracic Surgeons database. Innovations (Phila) 2014;9:10-5.

22. Deen SA, Wilson JL, Wilshire CL, et al. Defining the cost of care for lobectomy and segmentectomy: a comparison of open, video-assisted thoracoscopic, and robotic approaches. Ann Thorac Surg 2014;97:1000-7.

23. Swanson SJ, Miller DL, McKenna RJ Jr, et al. Comparing robot-assisted thoracic surgical lobectomy with conventional video-assisted thoracic surgical lobectomy and wedge resection: results from a multihospital database (Premier). J Thorac Cardiovasc Surg 2014;147:929-37.

24. Agzarian J, Fahim C, Shargall Y, et al. The Use of Robotic-Assisted Thoracic Surgery for Lung Resection: A Comprehensive Systematic Review. Semin Thorac
Cardiovasc Surg 2016;28:182-92.

25. Rocco G, Internullo E, Cassivi SD, et al. The variability of practice in minimally invasive thoracic surgery for pulmonary resections. Thorac Surg Clin 2008;18:235-47.

26. McKenna RJ. Complications and learning curves for video-assisted thoracic surgery lobectomy. Thorac Surg Clin 2008;18:275-80.

27. Yao F, Wang J, Yao J, Hang F, et al. Video-Assisted Thoracic Surgical Lobectomy for Lung Cancer: Description of a Learning Curve. J Laparoendosc Adv Surg Tech A 2017;27:696-703.

28. Meyer M, Gharagozloo F, Tempesta B, et al. The learning curve of robotic lobectomy. Int J Med Robot 2012;8:448-52.

29. Lee BE, Shapiro M, Rutledge JR, et al. Nodal Upstaging in Robotic and Video Assisted Thoracic Surgery Lobectomy for Clinical N0 Lung Cancer. Ann Thorac Surg 2015;100:229-33; discussion 233-4.

30. Toker A, Ozyurtkan MO, Kaba E, et al. Robotic anatomic lung resections: the initial experience and description of learning in 102 cases. Surg Endosc 2016;30:676-83.

31. Abbas AE. Robotic portal lobectomy, surgery through a virtual thoracotomy. J Thorac Dis 2017;9:2871-5.

32. Abbas A, Pompeo E, D'Amico TA. Video-Assisted Thoracoscopic and Robotic-Assisted Pulmonary Resections. In: Kaiser LR, Kron IL, Spray TL. editors. Mastery of cardiothoracic surger. Third. Philadelphia, Pennsylvania: Lippincott Williams \& Wilkins; 2014:108-15.

33. Abbas A, Dylewski MR, Lazzaro R. Complete PortAccess Robotic-Assisted Lobectomy Utilizing Three-Arm Technique Without a Transthoracic Utility Incision. In: Kim KC, editor. Robotics in General Surgery. New York: Springer-Verlag; 2014:69-85.

34. Cerfolio R, Louie BE, Farivar AS, et al. Consensus statement on definitions and nomenclature for robotic thoracic surgery. J Thorac Cardiovasc Surg 2017;154:1065-9.

35. Abbas AE. New nomenclature for robotic-assisted thoracic surgery also gets rid of RATS. J Thorac Cardiovasc Surg 2017;154:1070-1.

36. Chen X, Yang S, Guo W, et al. Robotic-assisted right middle lobectomy. AME Med J 2017;2:8.

37. Shulman RM, Abbas AE. The state of the art and future directions of robotic-assisted thoracic surgery. IJTCVS 2018;34:55.

38. Nisky I, Huang F, Milstein A, et al. Perception of stiffness in laparoscopy - the fulcrum effect. Stud Health Technol 
Inform 2012;173:313-9.

39. Li C, Han Y, Han D, et al. Robotic Approach to Combined Anatomic Pulmonary Subsegmentectomy: Technical Aspects and Early Results. Ann Thorac Surg 2019;107:1480-6.

40. Berguer R, Smith W. An ergonomic comparison of robotic and laparoscopic technique: the influence of surgeon experience and task complexity. J Surg Res 2006;134:87-92.

41. Abbas A, Kadakia S, Ambur V, et al. Intraoperative electromagnetic navigational bronchoscopic localization of small, deep, or subsolid pulmonary nodules. J Thorac Cardiovasc Surg 2017;153:1581-90.

42. Kodama K, Doi O, Higashiyama M, et al. Intentional limited resection for selected patients with T1 N0 M0 non-small-cell lung cancer: a single-institution study. J Thorac Cardiovasc Surg 1997;114:347-53.

Cite this article as: Mazzei M, Abbas AE. Why comprehensive adoption of robotic assisted thoracic surgery is ideal for both simple and complex lung resections. J Thorac Dis 2020;12(2):7081. doi: $10.21037 /$ jtd.2020.01.22
43. Matsumura Y, Yano M, Yoshida J, et al. Early and late recurrence after intentional limited resection for cT1aN0M0, non-small cell lung cancer: from a multiinstitutional, retrospective analysis in Japan. Interact Cardiovasc Thorac Surg 2016;23:444-9.

44. Wedmid A, Llukani E, Lee DI. Future perspectives in robotic surgery. BJU Int 2011;108:1028-36.

45. Kent M, Wang T, Whyte R, et al. Open, video-assisted thoracic surgery, and robotic lobectomy: review of a national database. Ann Thorac Surg 2014;97:236-42; discussion 242-4.

46. Wagner C, Stylopoulos N, Jackson P, et al. The benefit of force feedback in surgery: examination of blunt dissection. Presence Teleoperators Virtual Env 2007;16:252-62.

47. Brodie A, Vasdev N. The future of robotic surgery. Ann R Coll Surg Engl 2018;100:4-13. 